rev $\mid$ Psi

Revista de Psicología (UNLP)

https://revistas.unlp.edu.ar/revpsi

Reseña de libro

\title{
La teoría kleiniana desde los aportes de Hanna Segal: articulaciones teórico- clínicas sobre el desarrollo psicológico
}

\author{
Katja Sambeth ${ }^{1}$ \\ Correspondencia \\ katsamb@gmail.com \\ Filiaciones institucionales \\ ${ }^{1}$ Laboratorio de Investigaciones en Psicoanálisis y \\ Psicopatología, Facultad de Psicología, Universidad \\ Nacional de La Plata (Argentina)
}

\section{Datos del libro reseñado}

Segal, H. (1964/2008). Introducción a la obra de Melanie Klein (H. Friedenthal, Trad.). Buenos Aires: Paidos [124 pp; ISBN: 9789501240245$].$

\section{Desarrollo}

Desde sus inicios, Hanna Segal ha sido una figura destacada en el campo del psicoanálisis. Exiliada de Polonia durante el nazismo, completa sus estudios en medicina y emprende una formación psicoanalítica en Inglaterra. Más precisamente, allí integra la Sociedad Psicoanalítica Británica y comienza su análisis con Melanie Klein, destacada psicoanalista que contribuye a la formación del campo del análisis con niños, al considerar la actividad lúdica como un medio de expresión homologable a la producción onírica. Como manifestación del inconsciente, capaz de ser descifrado, propone que el juego es una vía de acceso a las fantasías inconscientes de los niños y, por ende, un equivalente de la asociación libre del adulto. En este

Editor

Nicolás Alessandroni | Facultad de Psicología, Universidad Autónoma de Madrid (España)

Sambeth, K. (2019). La teoría kleinian desde los aportes de Hanna Segal: articulaciones teórico-clínicas sobre el desarrollo psicológico. Revista de Psicología, 18(1), 97-103. doi: 10.24215/2422572Xe030
ISSN

2422-572X

Licencia

(c) Copyright: Sambeth, K. Se distribuye bajo una licencia de Cultura Libre CC-BY 4.0

Entidad editora

RevPsi es una publicación de la

Facultad de Psicología (Universidad

Nacional de La Plata, Argentina)

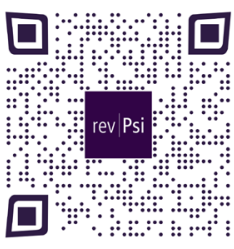

ACCESO ABIERTO DIAMANTE ə 
sentido, el fort-da que desarrolla Freud (1920/1989) es un precursor de la técnica de juego propuesta por Klein, a partir de la cual se escenifican las relaciones de objeto, las ansiedades y los mecanismos de defensa, presentes desde la temprana infancia. Es así que, apartándose de los presupuestos metapsicológicos freudianos y oponiéndose al psicoanálisis annafreudiano como tarea pedagógica, Klein se inscribe en la escuela inglesa con una nueva propuesta. El encuentro se desarrolló en un clima de entusiasmo y camaradería, aspecto que a lo largo de las distintas ediciones se ha convertido en un rasgo distintivo de esta convocatoria. Ello genera las condiciones para propiciar el debate y la profundización de saberes en torno al estudio de las complejidades que presenta el abordaje de las violencias, caracterizado por la participación activa, desde una perspectiva crítica y con especial interés en la producción de conocimiento situado.

La incursión de Hanna Segal en las formulaciones teóricas de esta autora permite reconocer sus destacados aportes a la comprensión conceptual, en torno a una articulación con la clínica. En otras palabras, su primer libro, Introducción a la obra de Melanie Klein (Segal, 1964/2008), se constituye en una pieza indispensable para tomar conocimiento de los desarrollos del psicoanálisis kleiniano, respecto a la conformación del yo, la existencia de relaciones objetales tempranas, la presencia de angustia a partir del nacimiento, entre otros conceptos.

Asimismo, ciertas producciones posteriores indican su interés por los procesos de simbolización en pacientes psicóticos y, además, en artistas. En torno a ellos, esboza su propuesta acerca de la obra de arte como acto de reparación ante la pérdida del objeto. Por lo tanto, siguiendo a Klein, se encamina hacia un análisis profundo de su obra y a la formulación de la experiencia estética como puesta en acto de las dos posiciones del desarrollo psicológico, planteadas por su antecesora.

Por lo mencionado, se persigue el propósito de relevar el carácter novedoso de la teoría kleiniana a lo largo de la historia del Psicoanálisis, no solamente en los contextos europeos, sino también en la posterior apropiación realizada en nuestro país. Más precisamente, son innegables las fundamentaciones teóricas kleinianas que sustentan variadas técnicas en Psicodiagnóstico y que hacen necesario su estudio. En este sentido, es propicio dar a conocer la producción de Hanna Segal, con el fin de visibilizar sus aportes en el campo de las ideas kleinianas a partir de su experiencia clínica. Además, esta obra se considera de gran pertinencia conceptual para aquellos que deseen tomar contacto con la perspectiva de la escuela inglesa e incursionar en la teoría de las relaciones objetales.

El libro se inicia con una presentación de la versión castellana, a cargo del psicoanalista argentino León Grinberg, quien destaca el importante esclarecimiento, realizado por Segal, de la complejidad teórica característica de la obra de Klein. Además, dado que el Psicoanálisis ha traspasado las fronteras del consultorio, Grinberg plantea la necesidad de que médicos, psiquiatras, psicólogos, sociólogos y estudiantes de diversas disciplinas, tomen conocimiento de esta teoría.

Posteriormente, se realiza un listado de los libros escritos por Klein, seguido de los 
agradecimientos de su discípula hacia sus pacientes, sus alumnos y otras figuras de gran ayuda. Luego de una introducción, se presentan ocho capítulos que ahondan en la teoría y se sostienen en casos clínicos propios de la autora. Se titulan de la siguiente manera: 1) Fantasía, 2) La posición esquizo-paranoide, 3) Envidia, 4) La psicopatología de la posición esquizo-paranoide, 5) La posición depresiva, 6) Defensas maníacas, 7) Reparación y 8) Los estados precoces del complejo de Edipo. Finalmente, un glosario reúne los términos técnicos cuyas definiciones, en base a la experiencia de la autora, son pertinentes para los estudiantes.

Tal como se menciona en la Introducción, el motivo de la obra se enmarca en la experiencia docente de Segal. El libro está basado en las clases brindadas a los alumnos de tercer año del Instituto Psicoanalítico de Londres. Como se ha señalado, con el fin de presentar las contribuciones de Melanie Klein a la teoría psicoanalítica, Segal decide ordenar los capítulos en torno al desarrollo psicológico del individuo. Comenzando por la temprana infancia, anticipa la conformación de dos posiciones o fases de dicho desarrollo, basadas en una configuración específica de relaciones objetales, ansiedades y defensas, persistente a lo largo de la vida (Klein, 1952/1988). En pos de aclarar que no constituyen meras etapas superadas unas a otras a lo largo del tiempo, Segal sostiene que el individuo puede oscilar, durante todo su ciclo vital, entre ambas posiciones: esquizo-paranoide y maníaco-depresiva.

La autora propone iniciar el recorrido teórico a partir de la consideración de las fantasías inconscientes, concepto de marcada importancia en la obra de Klein. En el capítulo 1, se definen como las expresiones mentales de los instintos que, por lo tanto, existen desde el comienzo de la vida. Por mediación del yo, se crean estas fantasías vinculadas con el establecimiento de relaciones objetales primitivas. En consecuencia, a diferencia de lo planteado por Freud, la autora propone que dicho grado de organización yoica está presente desde el nacimiento, momento traumático en que el bebé debe enfrentarse con el impacto de la realidad y con variadas experiencias de gratificación y frustración de sus deseos. Es por ello que hay una interrelación, sostenida en el tiempo desde los inicios, entre la fantasía y la realidad: una distorsiona a la otra, produciendo un impacto mutuo. Aquí también se destaca que las fantasías inconscientes permiten, por un lado, satisfacer impulsos instintivos prescindiendo de la realidad externa y, por otro, defenderse contra la realidad interna constituida por ciertos objetos introyectados con los que el yo mantiene relación. En torno a lo dicho, gracias a su experiencia clínica, la autora es capaz de articular dichas conceptualizaciones con los contenidos oníricos de sus pacientes.

Siguiendo estos lineamientos, el capítulo 2 presenta las características de la posición esquizo-paranoide, primera fase del desarrollo psicológico. Nuevamente se hace hincapié en que el yo está presente desde el nacimiento, motivo por el cual es capaz de sentir ansiedad, usar mecanismos de defensa y establecer primitivas relaciones objetales en la fantasía y en la realidad, en función de los polares instintos de vida y de muerte. Muy tempranamente, este yo desorganizado, lábil e inmaduro, tiene relación con dos objetos: el objeto primario y parcial, el pecho, está disociado en dos partes. Segal describe, a partir de dos casos clínicos, cómo la fantasía del objeto ideal 
se fusiona con experiencias gratificadoras de ser amado y amamantado por la madre externa real, mientras que la fantasía de persecución se fusiona con experiencias reales de privación y dolor. En torno a ello, en este capítulo se caracteriza la posición mencionada a partir de la presencia de una ansiedad paranoide, a partir de la cual se experimenta que el objeto persecutorio se introducirá en el yo y lo aniquilará junto con el objeto ideal. Contra esta ansiedad abrumadora, el yo desarrolla una serie de mecanismos de defensa. Por un lado, Segal señala la introyección y la proyección, que permiten mantener a los objetos persecutorios alejados de los objetos ideales, a la vez que se mantiene a ambos bajo control. Por otro lado, se detiene en la escisión, vinculada con la creciente idealización el objeto ideal, separado del objeto persecutorio. Seguidamente, es menester, según se afirma en esta sección, que la posición esquizo-paranoide dé lugar, en forma gradual, a la posición maníacodepresiva. Para ello, debe haber un predominio de experiencias buenas sobre las malas, las cuales permitan la disminución de la escisión del yo y de los objetos, ahora mayormente distinguidos.

Durante el capítulo 3, se realiza un recorrido por el concepto de envidia, considerada como la más temprana y primitiva emoción del bebé hacia el pecho que lo alimenta, objeto parcial, por sus cualidades como fuente de vida. Además, en esta sección, se la diferencia de los celos, cuyo objetivo es poseer al objeto total amado y excluir al rival, y de la voracidad, donde se intenta poseer todo lo bueno que pueda extraerse del objeto, sin considerar las consecuencias de su destrucción. Contrariamente, en la envidia, el propósito es ser tan bueno como el objeto, aunque, si esto se siente imposible, conlleva la intención de arruinar lo bueno que posee el objeto para suprimir la fuente de envidia. Una consecuencia de esta emoción temprana muy intensa, impregnada de instinto de muerte, es que no se puede mantener el proceso de escisión entre un objeto ideal y un objeto persecutorio. Segal sostiene que esta situación lleva a una confusión entre lo bueno y lo malo y afecta el curso de la posición esquizoparanoide, factor determinante de su psicopatología. Una vez más, articula dichas formulaciones con cierta fenomenología clínica, donde la envidia inconsciente yace en la raíz de las reacciones terapéuticas negativas y en los tratamientos interminables o fracasados.

Siguiendo esta temática, el capítulo 4 propone la psicopatología de la posición esquizoparanoide. La autora sostiene que, cuanto más perturbado esté el bebé, más se alejan sus experiencias de las que el observador adulto puede colegir por instrospección. No obstante, se propone que, en la enfermedad psíquica, se produce una regresión a los puntos de fijación de la psicosis presentes en una fase del desarrollo que ya posee rasgos patológicos en sí misma. Estos se centran en el predominio de experiencias malas sobre las buenas durante el fin de la posición esquizo-paranoide y en el uso patológico de la identificación proyectiva. En este caso, se postula que el niño enfermo siente que la parte de la realidad afectada por el proceso contiene objetos extraños cargados de enorme hostilidad, los cuales amenazan a un yo despojado y mutilado. Incorporando variados casos, Segal ilustra dicha situación a partir de, por ejemplo, un paciente adulto que vivencia una idealización precaria hacia la esposa, motivo por 
el cual le proyecta una gran magnitud de envidia y se vuelve persecutoria y hostil.

En el capítulo 5, se retoma lo dicho en torno al pasaje no patológico de la posición esquizo-paranoide a la maníaco-depresiva, donde el yo está integrado, su objeto ideal y sus impulsos libidinales son más fuertes que el objeto persecutorio y los impulsos agresivos, al tiempo que disminuyen la escisión y la proyección. En esta fase del desarrollo, se reconoce un objeto total: la madre provee experiencias buenas y malas al mismo tiempo, tiene una vida propia y ciertas relaciones con otras personas. Por lo tanto, Segal propone que, en este momento, el bebé descubre cuán desamparado está y cuánto depende de ella, lo cual intensifica los procesos de introyección. Esto trae consigo un cambio en las ansiedades, basadas en el temor a que sus propios impulsos hayan destruido al objeto amado. Como se ilustra en el caso clínico propuesto, aumenta su necesidad de poseer al objeto, incluso mientras lo odia, aspecto que denota la presencia de ambivalencia y que constituye y transforma el carácter del superyó. Por ende, el bebé está expuesto al duelo y la nostalgia por el objeto que se siente perdido, y a la culpa provocada por el sentimiento de que lo pierde por su propia destructividad. Sin embargo, la experiencia de depresión moviliza en el bebé el deseo de reparar a su objeto destruido, base de la creatividad y la sublimación. En este punto, se entiende la génesis de la formación de símbolos, donde el bebé inhibe sus instintos y los desplaza sobre sustitutos. Progresivamente, se plantea que los mecanismos psicóticos ceden lugar a mecanismos neuróticos: inhibición, represión y desplazamiento.

En torno a lo dicho, el capítulo 6 hace referencia al uso de las defensas maníacas durante la posición depresiva. Están destinadas primordialmente a impedir que se experiencie la realidad psíquica, a partir de una relación maníaca con los objetos caracterizada por una tríada de sentimientos. La autora propone que el control del objeto es una manera de negar la propia dependencia de él pero, al mismo tiempo, una forma de obligarlo a satisfacer una necesidad. El triunfo es la negación de sentimientos depresivos ligados a la valoración e importancia afectiva otorgada al objeto, mientras que el desprecio habilita sentir que el objeto no se merece que se sienta culpa por él, lo cual justifica continuar atacándolo.

Luego, en el capítulo 7, la autora afirma que la reparación es un mecanismo que se pone en juego cuando el bebé entra en posición depresiva y siente que ha destruido omnipotentemente a su madre. El deseo y la capacidad de restaurar al objeto, interno y externo, se basan en las actividades creadoras y en la capacidad del yo para conservar el amor y las relaciones a través de conflictos y dificultades. Por lo tanto, como se observa en las manifestaciones fenomenológicas destacadas, aquí se emplea aún más la prueba de realidad: el bebé observa con preocupación y ansiedad qué efecto tienen sus fantasías sobre los objetos.

Finalmente, en el complejo de Edipo temprano, se experiencia la relación edípica al comienzo de la posición depresiva. El capítulo 8 describe que, cuando el bebé se percata del vínculo libidinal existente entre sus padres, proyecta en ellos sus propios deseos libidinales y agresivos. Al percibir y fantasear que se brindan aquellas 
gratificaciones que él desea para sí, se origina intensa frustración, celos y envidia. Reacciona ante esta situación con sentimientos agresivos y los ataca, lo cual genera que se presente destruida a la pareja parental interna de la situación edípica temprana. Como se sostiene, para el varón y la niña, el primer objeto de deseos es el pecho de la madre, $y$ al padre se lo percibe primero como rival. No obstante, ante las ansiedades persecutorias y depresivas vivenciadas en relación con la madre y su pecho, el pene del padre se convierte en un objeto alternativo de deseo oral hacia el que la criatura se puede dirigir apartándose del pecho, tal como ilustran las presentaciones oníricas de los pacientes.

Respecto a las mencionadas teorizaciones, es menester hacer hincapié en la importancia de los aportes de Melanie Klein al Psicoanálisis. Por un lado, puede ser calificada como pionera en el trabajo psicoanalítico con niños, basado en el juego como medio de expresión simbólica de fantasías y deseos. Siguiendo a Freud (1914/1990) respecto de las escasas verbalizaciones de los niños como obstáculos al trabajo analítico, Klein (1932/1988) formula las bases de su técnica de juego como homologable a la regla fundamental, ya que, jugando, el niño habla y dice toda clase de cosas que tienen el valor de asociaciones genuinas. A su vez, considera que, en el juego, se ponen en funcionamiento las leyes del inconsciente, tal como sucede en los sueños. Este aspecto le permite sostener la posibilidad de analizar niños menores de seis años, refutando a los intentos antecesores, bajo la idea de que se logran elaborar situaciones traumáticas a partir del modo esencial de expresión de los niños pequeños, la acción.

Por lo referido, el juego estructura el psiquismo en tanto organizador del mundo interno y, además, es un indicador del desarrollo del funcionamiento mental. En otras palabras, tal como sostienen Luzzi y Bardi (2009), la ausencia de actividad lúdica en el niño es entendida como signo de la más severa patología infantil. Por lo tanto, las autoras sostienen que es imprescindible la precisión conceptual de Melanie Klein a la hora de pensar la psicoterapia con niños, con el fin de esclarecer los factores endógenos del desarrollo psicológico que direccionan un tratamiento. En consecuencia, Klein debe considerarse una autora de suma riqueza para la formación de analistas en clínica infantil.

Por otro lado, son indudables sus aportes respecto al área del Psicodiagnóstico, debido a que su teoría acerca de las relaciones objetales es fundamento de variadas técnicas proyectivas. Entre ellas, se puede destacar el Test de Relaciones Objetales de Phillipson [1955], la interpretación kleiniana del Cuestionario desiderativo de Bernstein [1960] y la sistematización de la Hora de Juego por Aberastury [1969].

En base a lo antedicho, cierto es que Hanna Segal ha contribuido en gran medida al esclarecimiento de la obra de Melanie Klein. En primer lugar, son destacables las ponderaciones realizadas a lo solicitado por los estudiantes, aspecto que le permite seleccionar, jerarquizar y ordenar sus ideas. En otras palabras, es su experiencia docente la que la incita a escribir este libro, la cual se ve favorecida por su capacidad didáctica y explicativa. En segundo lugar, como es sabido, la obra kleiniania presenta 
una complejidad significativa y, como es de suponer, no es sin Segal que ciertos aprendices incursionan en estos desarrollos teóricos psicoanalíticos. En tercer lugar, debe considerarse de gran relevancia la articulación entre teoría y práctica realizada. Tal como se ha evidenciado, todos los conceptos explicitados son ilustrados con variados casos clínicos, seleccionados por la autora a partir de su experiencia con niños y adultos. Siguiendo esta perspectiva, no sólo logran elucidar aún más las ideas teóricas de Klein, sino que son ejemplos de intervenciones analíticas realizadas en la clínica con pacientes psicóticos. Por lo tanto, es posible que esta obra contribuya a la comprensión de la interpretación y la transferencia como pilares del quehacer psicoanalítico.

En definitiva, es menester concluir que la claridad expositiva de la obra ofrece un gran valor a quienes desean incursionar en la teoría kleiniana. Asimismo, es útil para quienes busquen materiales clínicos, a la hora de favorecer y contribuir al desarrollo de sus espacios analíticos.

\section{Referencias}

Freud, S. (1920/1989). Más allá del principio del placer. En J. L. Etcheverry (Trad.), Obras completas. Tomo XVIII (pp. 1-62). Buenos Aires: Amorrortu.

Freud, S. (1914/1990). De la historia de una neurosis infantil. En J. L. Etcheverry (Trad.), Obras completas. Tomo XVII (pp. 1-112). Buenos Aires: Amorrortu.

Klein, M. (1932/1988). El psicoanálisis de niños Obras Completas. Tomo II. Buenos Aires: Paidós.

Klein, M. (1952/1988). Algunas conclusiones sobre la vida emocional del lactante. En Envidia $y$ Gratitud. Obras Completas. Tomo III. Buenos Aires: Paidós.

Luzzi, A. M. y Bardi, D. C. (2009). Conceptualización psicoanalítica acerca del juego de los niños. Punto de partida para una investigación empírica en psicoterapia. Anuario de Investigaciones, 16, 53-63.

Segal, H. (1964/2008). Introducción a la obra de Melanie Klein (H. Friedenthal, Trad.). Buenos Aires: Paidós. 